incidentally, can be expected to help resolve the issues around meeting the neglected needs of somatising patients.

1 Lepping $\mathrm{P}$, Palmstierna T, Raveesh BN. Paternalism $v$. autonomy - are we barking up the wrong tree? Br J Psychiatry 2016; 209: 95-6.

2 Wilkinson SR. Coping and Complaining: Attachment and the Language of Dis-ease (pp. 230-239). Brunner-Routledge, 2003.

Simon R Wilkinson, MD (Cantab), FRCPsych, Doctor, project manager, Akershus University Hospital, Lørenskog, Norway. Email: simonrwilkinson@gmail.com

doi: 10.1192/bjp.210.1.83b

Authors' reply: We are pleased that our paper has started a discussion about the ethics of autonomy for severely mentally ill patients. In our view, this has been long overdue. Both authors replying to our editorial ${ }^{1}$ have reasonable reflections, deepening our deliberations about the impact of any immediate reduction of autonomy on severely mentally ill patients and the balance with other ethical pillars that we all rely on in psychiatry.

Wilkinson raises the question of how attachment styles of the doctor could affect his or her communication style towards the patient, possibly increasing paternalism. It is an interesting point. It emphasises how paternalism could occur by the doctor being unaware of a 'paternalistic' communication and decision style. This is a relevant comment regarding how we as doctors interact with our patients, creating a more or less 'coercive' style.

Crichton, on the other hand, elaborates on the issue of how autonomy is in fact already restricted for patients. We acknowledge this aspect as relevant; however, we would equally like to stress that autonomy is not automatically more important than other ethical pillars. In our opinion, there is a danger in overemphasising immediately expressed autonomy in every situation, as it risks compromising both future autonomy and other pillars of medical ethics. We merely discuss the balance between autonomy and the other central pillars of medical ethics in medicine, and particularly in psychiatry. Crichton's call for consideration of the already limited autonomy is justified, but this should be a starting point for a more detailed discussion. Patients may understand their situation and choices, but are not autonomous unless they are able to form value judgements about their reasons for choosing treatment. So stating that autonomy is limited is a judgement which needs to be carefully examined from an ethical point of view. In addition, autonomy will be interpreted differently in various social, religious, judicial, political, philosophical and medical contexts.

We are aware that autonomy is restricted for all of us by several components, and that action should be taken to increase it. But we would like to argue that, in order to increase patients' autonomy over time, there is a need to act upon all pillars of medical ethics. We argue that we should consider that the immediate choices expressed by the patient may occasionally have to be balanced with best interests decisions, both to preserve the integrity of the other pillars of medical ethics (providing safety, protection, treatment) and to promote future autonomy of the patient. Furthermore, we strongly believe that the immediate request for autonomous decision-making expressed by a severely disordered patient should not be a simple excuse to neglect other ethical considerations, just because it is the easiest way to proceed. In our view, this would be a dangerous road to follow, although anecdotal evidence suggests that it is already occasionally happening. It demands nothing of us as psychiatrists, but could have devastating consequences for patients in the end. It could undermine all the ethical pillars we regard as important, not only for the well-being of the patient but also for the patient's future ability to make true autonomous decisions about his or her life. We argue that taking a stand to evaluate all the ethical pillars of medical ethics is the right way to go, but it is also a demanding way along 'a long and winding road'.

1 Lepping $\mathrm{P}$, Palmstierna T, Raveesh BN. Paternalism v. autonomy - are we barking up the wrong tree? Br J Psychiatry 2016; 209: 95-6.

Peter Lepping, PETER.LEPPING@wales.nhs.uk; Tom Palmstierna Tom.Palmstierna@ki.se; Bevinahalli N. Raveesh, raveesh6@yahoo.com

doi: 10.1192/bjp.210.1.84

\section{Measuring outcomes of mindfulness interventions}

Wong and colleagues are to be congratulated for the large scale randomised controlled trial on mindfulness-based cognitive therapy versus group psychoeducation for people with generalised anxiety disorder. ${ }^{1}$ We have studied mindfulness awareness practice (MAP) amongst elderly individuals in an open-label study ${ }^{2}$ and more recently in a randomised controlled trial (ClinicalTrials.gov registration NCT02286791) and would like to share our experiences. Both studies involved community-living elderly people, with the second study involving individuals with mild cognitive impairment.

Wong et al highlight the use of self-reported questionnaires as one of the limitations of the study. We do agree and suggest that measurement of 'psychobiomarkers' may be the solution. Self-reports are useful for estimating psychological efficacy with task-based or behavioural approaches. ${ }^{3}$ But many of the mental changes achieved even in short-term meditative practice are better measured through the physiological changes associated with achieving mental balance (conative, attentional, cognitive and affective $)^{4}$ in contemplative practices. These are at the structural, cellular and biochemical level, and in preliminary findings in our study, changes in functional brain activity, neuropsychological tests, telomere lengths and oxidative stress markers were noted after 12 weeks of mindfulness practice (manuscript in preparation).

Like Wong and his colleagues, we too noted similar improvements in the control group which was provided weekly health education talks. We hold similar views that these resulted from the benefits of the group activity and the time and attention provided. Despite improvements in the control group, the changes were more significant in the MAP intervention arm.

Until we have identified the best biological measurement tools to identify the changes brought about by meditative practices, it may be too soon to dismiss mindfulness-based interventions for our patients. We agree that specific groups of patients with targeted needs would be better suited for mindfulness-based clinical programmes, and the challenge would be in identifying these patients and conditions. Would the authors comment on the implications of cultural factors and religious and spiritual beliefs in the usefulness of mindfulness interventions?

1 Wong SYS, Yip BHK, Mak WWS, Mercer S, Cheung EYL, Ling CYM, et al. Mindfulness-based cognitive therapy $v$. group psychoeducation for people with generalised anxiety disorder: randomized controlled trial. Br J Psychiatry 2016; 209: 68-75.

2 Rawtaer I, Mahendran R, Yu J, Fam J, Feng L, Kua EH. Psychosocial interventions with art, music, Tai Chi and mindfulness for subsyndromal depression and anxiety in older adults: a naturalistic study in Singapore. Asia Pac J Psychiatry 2015; 7: 240-50.

3 Kemeny ME, Foltz C, Cavanagh JF, Cullen M, Giese-Davis J, Jennings P, et al. Contemplative/emotion training reduces negative emotional behavior and promotes prosocial responses. Emotion 2012; 12: 338-50. 
4 Wallace BA, Shapiro SL. Mental balance and well-being: building bridges between Buddhism and Western psychology. Am Psychol 2006; 61: 690-701.

Rathi Mahendran, Senior Consultant Psychiatrist and Associate Professor, Department of Psychological Medicine, National University of Singapore. Email: medrm@nus.edu.sg; Ee Heok Kua, Senior Consultant Psychiatrist and Professor Department of Psychological Medicine, National University of Singapore. Email: pcmkeh@nus.edu.s

doi: 10.1192/bjp.210.1.84a

Author's reply: We thank Mahendran and colleagues for their comments and suggestions on our published study ${ }^{1}$. We agree that it would be helpful to have more objective measurements for attention, affective or cognitive changes which might not have been captured by self-reported questionnaires. For the potential confounding effects of religious or cultural beliefs on outcomes, we have only collected data on religious beliefs in our study, with $36.7 \%$ of participants being Christians/Catholics, 10.2\% being Buddhists/Taoists, and $53.1 \%$ being non-religious. We did not find a significant effect of religious beliefs on our psychological outcomes in this study.

1 Wong SYS, Yip BHK, Mak WWS, Mercer S, Cheung EYL, Ling CYM, et al. Mindfulness-based cognitive therapy $v$. group psychoeducation for people with generalised anxiety disorder: randomized controlled trial. Br J Psychiatry 2016; 209: 68-75.

Samuel Yeung Shan Wong, School of Public Health and Primary Care, Chinese University of Hong Kong. Email: yeungshanwong@cuhk.edu.hk

doi: 10.1192/bjp.210.1.85 